\title{
A NOTE ON GRADIENT MAPPINGS
}

\author{
E. H. ROTHE
}

1. Introduction. Let $E$ be a real Banach space, and $V$ a (proper or improper) subset of $E$. Let $D(x, h)$ be a function defined for $x$ in $V$ which for fixed $x$ is a linear continuous functional on $E$ in the variable $h$, in other words, let $D(x, h)$ define a map of $V$ into the space $E^{*}$ conjugate to $D$. Let $E$ and $E^{*}$ have the topologies induced by their respective norms. The map $D(x, h)$ is called completely continuous if it is continuous and if the image of each bounded subset of $V$ has a compact closure in $E^{*}$. It is said to be a gradient mapping if there exists a scalar function $I(x)$ such that $D(x, h)$ is the Fréchet differential of $I(x)$ at the point $x$. For the motivation of this terminology and for details, we refer the reader to [6] or [7]. It is known that there is a close connection between the complete continuity of the gradient on the one hand and the weak continuity or related properties of the scalar $I(x)$ on the other hand [6, Theorems 3.2 and 3.3]. Further literature is quoted in [7, footnote 11]; see also [2] and [3]. In particular, it is known [6, Theorem 3.3] that for convex $V$ the complete continuity of $D(x, h)$ implies the following property of the scalar $I(x)$ : to each positive $\eta$ there corresponds a finite number of elements $l_{i}(i=1,2, \cdots, N)$ of $E^{*}$ such that the inequalities

$$
\left|l_{i}(h)\right|<\eta\|h\| / 2 \quad(i=1,2, \cdots, N)
$$

imply

$$
|I(x+h)-I(x)|<\eta\|h\|, \quad h, x+h \text { in } V .
$$

For the case where $E$ is a Hilbert space, the converse of this theorem was stated and proved in [6]. The reason for the restriction was as follows: Hilbert spaces are the only Banach spaces (of dimension at least 3 ) with the property that there exists a projection of norm 1 on every closed linear subspace (see [5]), and such projections were used in the proof given in [6].

However, it will be seen in the present note, that for the purpose at hand it is not necessary to have projections of norm 1 (or of uniformly bounded norm) on all closed linear subspaces; rather, it will be sufficient that such projections exist on a large enough collection of subspaces. To be more precise we introduce the following concept.

Received by the editors October 7, 1958 and, in revised form, February 6, 1959.

1 The paper was written while the author was recipient of a John Simon Guggenheim Memorial Fellowship. 
Definition 1.1. Let $E$ be a Banach space and $E^{*}$ the space conjugate to $E$. We say that $E$ has property $P$ if there exists a set $\left\{f_{i}\right\}(i=1,2, \cdots)$ of linearly independent elements of $E^{*}$ and a positive number $M$ with the following two properties: the finite linear combinations of elements of $\left\{f_{i}\right\}$ are dense in $E^{*}$ (that is, $\left\{f_{i}\right\}$ is a fundamental set in $E^{*}$ in the sense of Banach [1, p. 58]), and if $N_{i}=\left\{x \in E \mid f_{i}(x)=0\right\}$, then for each integer $n$ there exists a projection of norm at most $M$ on the intersection $\bigcap_{i}^{n} N_{i}$.

In $\$ 2$ we shall show that the converse theorem in question is true under the assumption $P$; that is we shall prove the following

Theorem 1.1. Let $E$ be a Banach space with property P. Let $I(x)$ be a scalar defined in a convex subset $V$ of $E$ with the following two properties: $I(x)$ has a Fréchet differential which is continuous in $x$, and corresponding to each positive $\eta$ there exists a finite number of elements $l_{1}, l_{2}, \cdots, l_{n}$ of $E^{*}$ such that

$$
|I(x+h)-I(x)|<\eta\|h\| \quad(h, x+h \text { in } V)
$$

for all $h$ for which

$$
\left|l_{i}(h)\right| \leqq \eta\|h\| \quad(i=1,2, \cdots, n) .
$$

Then $D(x, h)$ is completely continuous.

$\$ 3$ deals with conditions that are sufficient for a Banach space $E$ to have property $P$. In particular, every reflexive Banach space with a base will be seen to have property $P$.

2. Proof of Theorem 1.1. We first recall some properties of an arbitrary Banach space $E$. Let $l_{1}, l_{2}, \cdots, l_{p}$ be $p$ linearly independent elements of $E^{*}$, and let $N=\left\{x \in E \mid l_{i}(x)=0, i=1,2, \cdots, p\right\}$. Moreover let $a_{1}, a_{2}, \cdots, a_{p}$ be any set of $p$ elements of $E$ which are linearly independent $\bmod N$. (This means that $\sum_{i=1}^{n} a_{i} \gamma_{i} \in N$ implies that the real numbers $\gamma_{i}$ are all 0 .) Finally let $E^{p}$ be the space spanned by the $a_{i}$. Then each $x$ in $E$ admits a unique decomposition

$$
x=x_{1}+n \quad\left(x_{1} \in E^{p}, n \in N\right),
$$

and, on account of the linear independence of the $l_{i}$, the determinant of the $l_{i}\left(a_{j}\right)$ is different from zero. It is easily seen that this latter fact implies the existence of a base $b_{1}, b_{2}, \cdots, b_{p}$ of $E^{p}$ which is "orthogonal," that is, for which

$$
l_{i}\left(b_{j}\right)=\delta_{i j} \quad(i, j=1,2, \cdots, p),
$$

where $\delta_{i j}$ is the Kronecker symbol. If then $l(x)$ is an arbitrary linear 
continuous functional on $E$, one obtains almost immediately the representation

$$
l(x)=\sum_{j=1}^{p} l_{j}(x) l\left(b_{j}\right)+l(n),
$$

(apply $l$ to (2.1) after $x_{1}$ has been expressed in terms of the $b_{j}$, and use (2.2)). With the notation of this paragraph, the following lemma is an immediate consequence of the representation (2.3); it may be considered as a generalization of the well-known fact that any $l$ vanishing on $N$ is a linear combination of the $l_{i}$.

Lemma 2.1. Let $l(x)$ have the property that there exists a positive number $\eta$ such that

$$
|l(y)|<\eta\|y\| \quad \text { for all } y \text { in } N .
$$

Then, for all $x$ in $E$,

$$
l(x)=\sum_{i=1}^{p} \alpha_{i} l_{i}(x)+R(x)
$$

with suitable constants $\alpha_{i}$ and with

$$
|R(x)| \leqq \eta\|n\|,
$$

where $n$ is the element of $N$ appearing in the decomposition (2.1) of $x$.

In addition to Lemma 2.1 we shall need the following

LemMa 2.2. If the assumption in Lemma 2.1 holds, and if there exists a projection $\pi$ of $E$ on $N$ of norm at most $M$, then the representation (2.5) holds with

$$
|R(x)| \leqq M \eta\|x\| .
$$

Proof. Since the elements $b_{i}$ are linearly independent $\bmod N$, the elements $b_{i}^{\prime}=b_{i}-\pi b_{i}$ have the same property. If then ' $E^{p}$ is the space spanned by the $b_{i}^{\prime}$, we obtain instead of (2.1) the decomposition

$$
x=x_{1}^{\prime}+n^{\prime} \quad\left(x_{1}^{\prime} \in E^{\prime} E^{p}, n^{\prime} \in N\right) .
$$

It is easily verified that $x_{1}^{\prime}=x-\pi(x)$ and $n^{\prime}=\pi(x)$, and consequently, by assumption,

$$
\left\|n^{\prime}\right\| \leqq M\|x\| .
$$

If we now apply Lemma 2.1 to the new decomposition (2.8), we have to replace $n$ by $n^{\prime}$ in (2.6). The inequality thus obtained together with (2.9) proves (2.7). 
We now turn to the proof of Theorem 1.1. We have to establish the complete continuity of the gradient mapping $D(x, h)$. For this purpose we may without loss of generality assume that $V$ is bounded. Therefore, by [6, Lemma 3.2] it will be sufficient to prove that, corresponding to a given $\epsilon>0$, there exists a mapping $D^{\prime}(x, h)$ of $V$ into $E^{*}$ with the following two properties: the image of $V$ under $D^{\prime}$ is contained in a finite-dimensional subspace of $E^{*}$, and

$$
\left|D(x, h)-D^{\prime}(x, h)\right| \leqq \epsilon\|h\| .
$$

We first choose, corresponding to the given $\epsilon$,

$$
\eta=\epsilon / M,
$$

where $M$ is the number appearing in Definition 1.1. Corresponding to $\eta$, there exist by assumption elements $l_{i}$ of $E^{*}$ such that (1.4) implies (1.3). Now, by Definition 1.1 , the set $\left\{f_{i}\right\}$ is fundamental in $E^{*}$. Consequently there exist finite linear combinations $f_{i}^{\prime}$ of elements of the set $\left\{f_{i}\right\}$ such that $\left\|l_{i}-f_{i}^{\prime}\right\|<\eta$, in other words, such that

$$
\left|l_{i}(h)-f_{i}^{\prime}(h)\right|<\eta\|h\| \quad(i=1,2, \cdots, n) .
$$

Now let the integer $p$ be such that the set $f_{1}, f_{2}, \cdots, f_{p}$ contains all elements of $\left\{f_{i}\right\}$ which occur in the linear combinations $f_{i}^{\prime}(i=1,2, \cdots, n)$, and let $N=\left\{h \mid f_{i}(h)=0, i=1,2, \cdots, p\right\}$. Then, for $h$ in $N$, all $f_{i}^{\prime}(h)$ are zero, and (2.12) shows that (1.4) holds for such $h$; by assumption this implies (1.3). But from (1.3) together with the definition of the Fréchet differential, one concludes easily (see $[6$, p. 430]) that

$$
|D(x, h)| \leqq \eta\|h\|
$$

for all $h$ in $N$. We now may apply Lemma 2.2, since by Definition 1.1 there exists a projection of $E$ on $N$ of norm at most $M$. In the present notation, we thus obtain from (2.5), (2.7), and (2.11)

$$
D(x, h)=\sum_{i=1}^{p} \alpha_{i} f_{i}(h)+R(h), \quad|R(h)| \leqq M \eta\|h\| \leqq \epsilon\|h\| .
$$

This shows that, with the definition

$$
D^{\prime}(x, h)=\sum_{i=1}^{p} \alpha_{i} f_{i}(h)
$$

$D^{\prime}(x, h)$ satisfies the two requirements formulated at the beginning of this proof. 
3. Sufficient conditions for property $P$. Let $E$ be a Banach space with a base $\left\{b_{i}\right\}$. Then there exists a unique sequence $\left\{f_{i}\right\}$ of elements of $E^{*}$ such that, for every element $x$ of $E$,

$$
x=\sum_{i=1}^{\infty} b_{i} f_{i}(x)
$$

[1, Chapter VII, §3]. We now make the assumption that the set $\left\{f_{i}\right\}$ is fundamental in $E^{*}$, and we claim that then $E$ has property $P$. Indeed, if $N^{p}=\left\{x \in E \mid f_{i}(x)=0\right.$ for $\left.i=1,2, \cdots, p\right\}$, then $N^{p}$ is the space spanned by $b_{p+1}, b_{p+2}, \cdots$. It follows from Banach's results $\left[1\right.$, p. 111] that the map $E \rightarrow N^{p}$ mapping the point (3.1) of $E$ into the point

$$
\sum_{i=p+1}^{\infty} b_{i} f_{i}(x)
$$

is a projection with a norm admitting a bound independent of $p$. This shows that $E$ has property $P$.

It follows in particular that every reflexive Banach space with a base has property $P$. For in such spaces, our assumption that the set $\left\{f_{i}\right\}$ is fundamental is satisfied; this follows from [4, Lemma 1, p. 70 together with Theorem 3, p. 71].

For reflexive Banach spaces with a base, Citlanadze [2] stated without proof some propositions related to Theorem 1.1. In a later paper [3, Theorem 1] he proved such a theorem in the more special case of an $L_{p}$ space $(p>1)$.

\section{BIBLIOGRAPHY}

1. S. Banach, Théorie des opérations linéaires, Warszawa, 1932.

2. E. S. Citlanadze, Some problems of nonlinear operators and the calculus of variations in spaces of Banach type, Uspehi Mat. Nauk N. S. vol. 5 (1950) pp. 141-142 (Russian).

3. - The method of orthogonal trajectories for nonlinear operators of variational type in the space $L_{p}$, Akad. Nauk Gruzin. SSR. Trudy Tbiliss. Mat. Inst. Razmadze vol. 20 (1954) pp. 245-278 (Russian); Amer. Math. Soc. Translations ser. 2 vol. 5 (1957) pp. 305-333.

4. M. M. Day, Normed linear spaces, Ergebnisse der Mathematik und ihrer Grenzgebiete, Neue Folge, Heft 21, Springer, 1958.

5. S. Kakutani, Some characterizations of Euclidean space, Jap. J. Math. vol. 15 (1939) pp. 93-137.

6. E. H. Rothe, Gradient mappings and extrema in Banach spaces, Duke Math. J. vol. 15 (1948) pp. 421-431.

7. - Gradient mappings, Bull. Amer. Math. Soc. vol. 59 (1953) pp. 5-19.

The University of Michigan 